\title{
STOCHASTIC SIMULATION OF CHARGED PARTICLE TRANSPORT ON THE MASSIVELY PARALLEL PROCESSOR
}

\author{
James A. Earl \\ Department of Physics and Astronomy \\ University of Maryland, College Park MD 20742
}

\begin{abstract}
Computations of cosmic-ray transport based upon finite-diference methods are afflicted by instabilities, inaccuracies, and artifacts. To avoid these problems, we have developed a Monte Carlo formulation which is closely related not only to the finite-difference formulation, but also to the underlying physics of transport phenomena. Implementations of this approach are currently running on the Massively Parallel Processor at Goddard Space Flight Center, whose enormous computing power overcomes the poor statistical accuracy that usually limits the use of stochastic methods. These simulations have progressed to a stage where they provide a useful and realistic picture of solar energetic particle propagation in interplanetary space.
\end{abstract}

Keywords: Cosmic-rays, Particle Transport, Interplanetary Medium.

\section{INTRODUCTION}

The diffusion idealization, which has been almost universally invoked in discussions of cosmic-ray transport is easy to treat analytically. However, many observed phenomena give clear evidence for non-diffusive effects. One example is the so-called "scatter free" propagation of kilovolt solar electrons (Ref. 1), which is inconsistent with diffusion, but which can readily be interpreted in terms of a coherent mode of propagation. This mode is novel, but it is just a manifestatation in a dynamic situation of non-diffusive effects similar to those considered in the steady-state by classical transport theory (Ref. 2). Although these effects have been described analytically in References 3 and 4 , the theory is very complicated. Consequently, there is a need for reliable numerical computations which bypass these complexities and yield concrete results suitable for comparison with observations. This paper describes computations of charged particle transport along a large-scale guiding magnetic field $\mathrm{B}$ whose spatial variations are characterized by the focusing length $\mathrm{L}$, which is defined by

$$
\frac{1}{L}=-\frac{1}{B} \frac{\partial B}{\partial z}
$$

where $\mathrm{z}$ is distance parallel to the guiding field. Particle trajectories are scattered by small-scale random magnetic fields whose effect is described by the mean free path $\lambda$. Note that the magnetic fields are visualized as static and that there is no interaction among particles in an extremely tenuous distribution of charged particles. This situation differs from those considered by plasma physics, but it is closely analogous to those treated by classical transport theory.
Over the past three years the computations have evolved through simulations of rectilinear transport along a constant guiding field, which were presented at the First Symposium on Massively Parallel Scientific Computation (Ref. 5), and of focused transport with constant focusing length and mean free path (Ref. 6 ), to the present simulations, which allow arbitrary variations of the focusing length and mean free path. In the interplanetary context, this formulation includes all important effects except those of convective motion of the background medium, which significantly affect the slow variations of cosmic-ray modulation, but which play a minor role during the rapid evolution of solar particle events. It includes the two essential aspects of charged particle transport. These are a strong inhibition of transport perpendicular to the guiding field and a strong anisotropy of the pitch-angle scattering by random fields.

\section{TRANSPORT EQUATIONS}

Under the circumstances outlined above, particle transport is described by

$$
\frac{\partial h}{\partial s}+\mu \frac{\partial h}{\partial z}=\frac{\partial}{\partial \mu} \psi e^{G} \frac{\partial}{\partial \mu} h e^{-G}
$$

in which $h$ is the number of particles per unit distance parallel to the guiding field, $\mu$ is the cosine of the pitch-angle. The parameter $s=\mathrm{Vt}$, where $\mathrm{V}$ is particle velocity, plays the role of a temporal variable. The Fokker-Planck coefficient of pitch-angle scattering is given by

$$
\psi=\frac{3\left(1-\mu^{2}\right)|\mu|^{q-1}}{2 \lambda(2-q)(4-q)}
$$

where $\mathrm{q}$ is an index that measures the anisotropy of scattering (Ref. 7). The function $G$ that appears in Equation 2 is defined in terms of $\psi$ by

$$
G(\mu)=\frac{1}{L} \int_{0}^{\mu} \frac{1-\nu^{2}}{\psi(\nu)} d \nu=\frac{(4-q)}{3} \frac{\lambda}{L} \mu|\mu|^{1-q}
$$


In the discrete formulation, the continuous variables are replaced by a three-dimensional grid whose spacings are $\Delta z, \Delta \mu$, and $\Delta s$, and the derivatives appearing in equation (1) are replaced by their finite-difference analogs. The effect of these replacements is best described in terms of the particles flowing in and out of a cell, whose dimensions are $\Delta \mu$ and $\Delta z$, during a temporal increment $\Delta s$. These flows are illustrated in Figure 1 , where the integer $M$ refers to the pitch angle, and the integer $Z$ refers to the distance $z$. Here, particles passing through the top and bottom of the box are those whose pitch angle changes because of scattering, and particles passing through the sides are those whose distance changes as a result of their motion parallel to the guiding field. The flows due to scattering can be described in terms of coefficients pM and $\mathrm{qM}_{\mathrm{M}}$, which characterize the flows out of box

$M$ toward larger and smaller values of $\mu$, respectively, and whose numerical values can be derived from Equation 2. The difference equations that correspond to Figure 1 can be solved by standard numerical methods, but these methods are difficult to implement and subject to subtle errors, which are discussed in Reference 8.

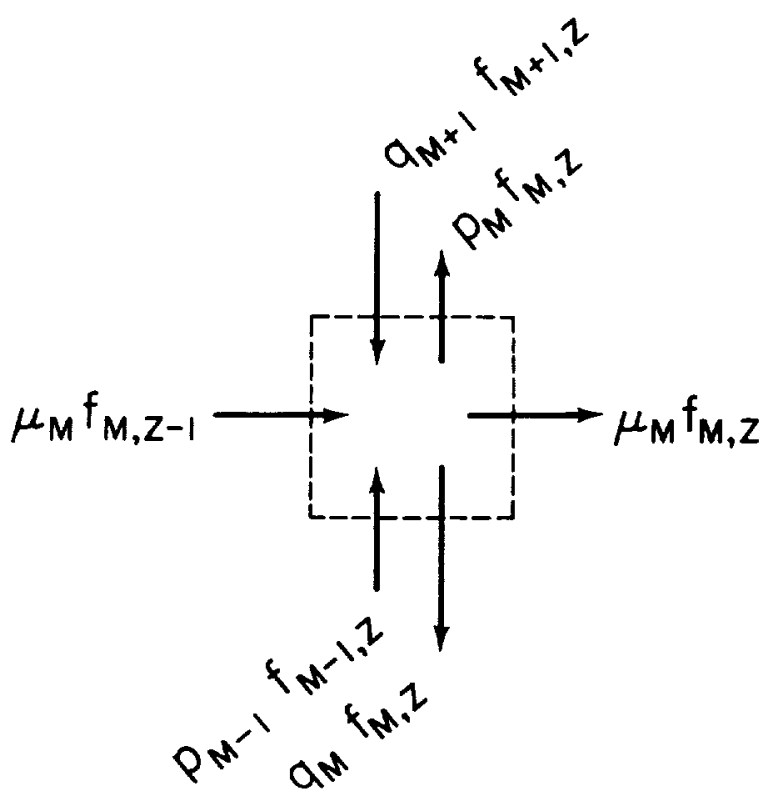

Figure 1. The number of particles in each cell of the $\mu-z$ plane changes, because scattering causes particles to flow in and out through the top and bottom boundaries, while motions in space cause them to flow through the vertical boundaries.

In the Monte Carlo formulation, the random history of a large number of particles is followed under the assumption that the coefficients $p$ and $q$ can be interpreted as probabilities in each temporal step that $\mu$ will change by $\Delta \mu$ toward more forward or more backward directions, respectively. Obviously, this defines $1-p-q$ as the probability that a pitch angle will not change. After their pitch angles are updated in each step, the particles move a distance $\mu \Delta$ s.

In Figure 2, the transition probabilities are plotted as a function of $\mu$ for strongly anisotropic scattering, $\mathrm{q}=1.8$, similar to that occuring in interplanetary space and for strong focusing, $\lambda / \mathrm{L}=5$. These probabilities exhibit the same gross features as the Fokker Planck coefficient, but there is a significant assymetry such that $p$ is consistently larger than $q$. This leads to the systematic drift toward forward directions that is expected as a result of focusing. Note that the probability of going backward through $\mu=0$ is only $0.13 \%$ in this example. This leads to a coherent mode of transport that is very different from diffusion, for any particle that reaches the forward hemisphere has a very small chance of going backward again.

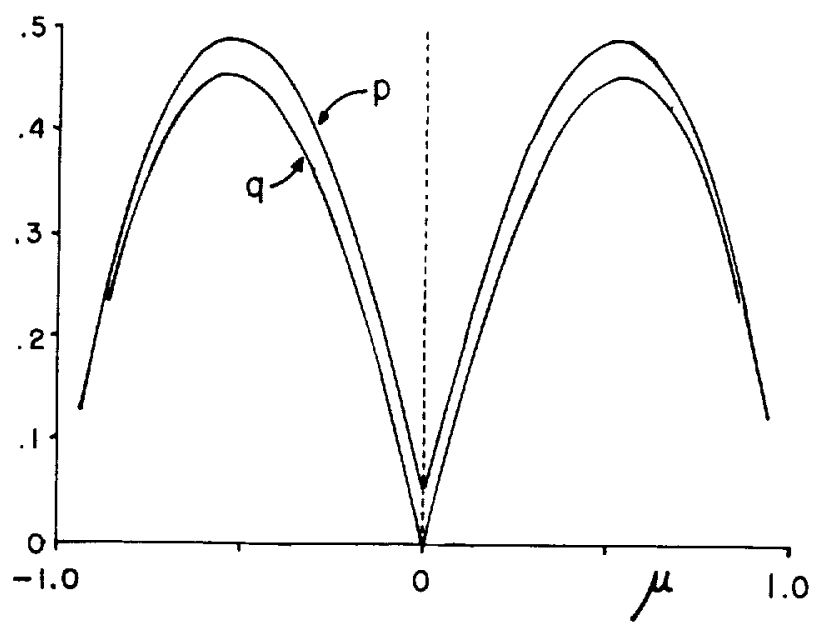

Figure 2. Forward and backward transition probabilites plotted as a function of pitch-angle cosine.

\section{THE ALGORITHM}

To implement the Monte Carlo scheme outlined above, each particle was assigned an integer distance and an index $M$ corresponding to $\mu$ that lies between 0 and 8 . Because these parameters occupy only three bytes for each particle, there was plenty of storage for several parallel arrays of particles. Consequently, the results given below are based on 32 arrays which contained $32 * 16384=524288$ particles. The fate of the particles was determined by a single parallel array of random integers ( ranging from -32767 to +32767 ) that was updated frequently. To implement changes in the pitch-angle cosine, forward and backward integers were assigned to each particle, according to its pitch angle and distance, in such a way that the probability of the current random number being larger than the integer is the corresponding forward or backward probability analogous to those poltted in Figure 2. Then the angular index was incremented for those particles whose current random integer was positive and greater than the forward integer, and decremented for those whose random integer was negative and less than the backward integer with its sign reversed. This approach satisfies the basic requirement that the probabilities of incrementing, decrementing and leaving unchanged the pitch-angle must add to unity. After the pitch-angles had been updated, each particle's distance was incremented. When the desired number of temporal steps had been carried out, particles were binned according to distance and pitch-angle. 


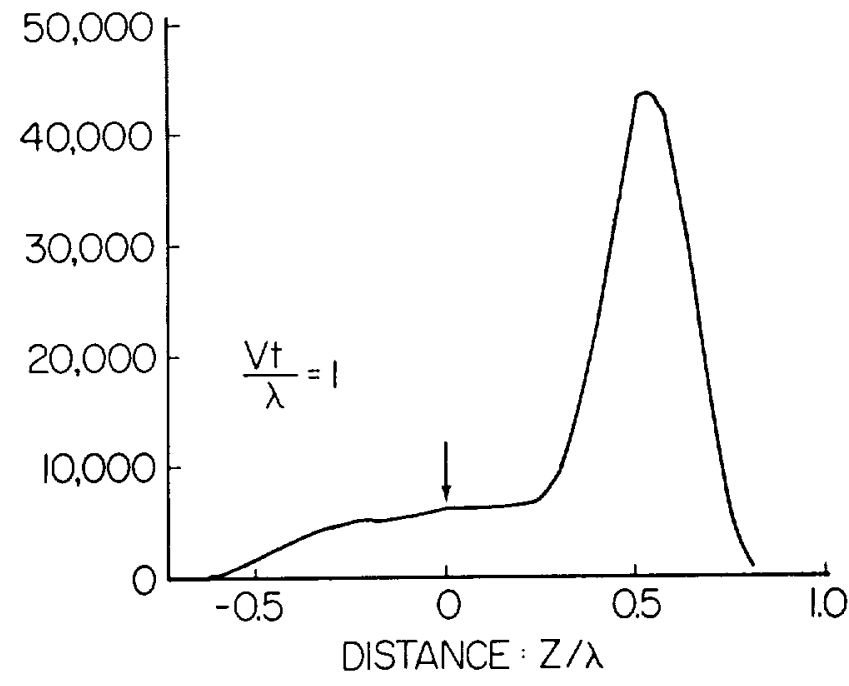

Figure 3. Density profile for rectilinear transport after a collimated injection at the arrow.

\section{SPATIAL AND TEMPORAL PROFILES}

To set the stage for the simulations of interplanetary transport that are the main subject of this paper, it is useful to consider first the case of rectilinear transport, in which the guiding field is constant, and focusing does not occur. Figure 3 presents results obtained from the MPP as plots of the total number of particles in each distance bin. This sum over pitch-angles is a measure of the isotropic particle density. Because the total number of particles was large, statistical errors are small and, consequently, are not shown explicitly. However, slight irregularities in some parts of the curves give an indication of their magnitude.

This density profile describes a situation very shortly after the injection of a collimated beam of when the particles have had time to move a maximum distance of only one mean free path. The curve exhibits two features: an intense localized peak at the right and a broad wake spread on both sides of the arrow which indicates the point of injection. Qualitatively, the peak appears because particles become nearly uniformly distributed in the forward hemisphere, while very few particles penetrate to the backward hemisphere through the region of weak scattering at $\mu=0$. This means that the particles in the forward hemisphere move with nearly the same average velocity parallel to the field, but statistical fluctuations in individual velocities give rise to a peak centered around the average displacement. Such features are designated as coherent pulses. They decay exponentially as particles slowly escape into the backward hemisphere and join the wake.

In the spiral pattern of the interplanetary magnetic field, the focusing assymetry that appears in Figure 2 tends to keep particles in the forward hemisphere. Consequently, the coherent pulse is long lived and intense relative to the wake. Moreover, the assymetry becomes very pronounced near the sun, for the ratio $\lambda / L$ varies approximately as $1 / r$, where $r$ is distance from the sun.

To illustrate these effects, Figure 4 shows snapshot profiles of number of particles vs. $r$ for four equally spaced times after injection into a model interplanetary field at $r=0$. These profiles describe a moving pulse whose width increases as it moves out, which is the qualitative behavior expected. However, the exact evolution of this width and the deceleration of the peak, which is evident from a careful examination of the figure, are details which crucially affect observations, but which are not adequately described by the analytic theory of focused transport.

In contrast with the rectilinear profile illustrated by Figure 2 , the wake is virtually invisible in all of the four profiles. As was discussed above, this enhancement of the coherent mode is a consequence of focusing.

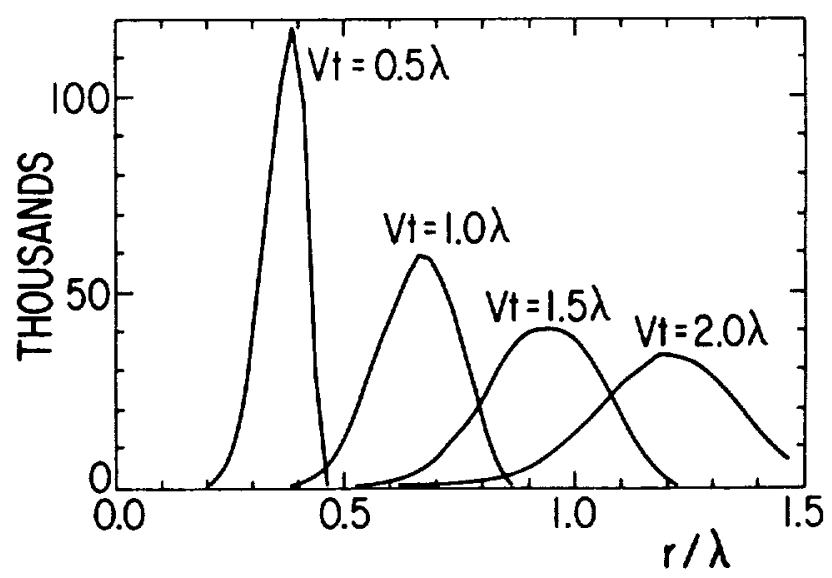

Figure 4. Profiles of isotropic particle density vs. distance from the sun for four different times after injection.

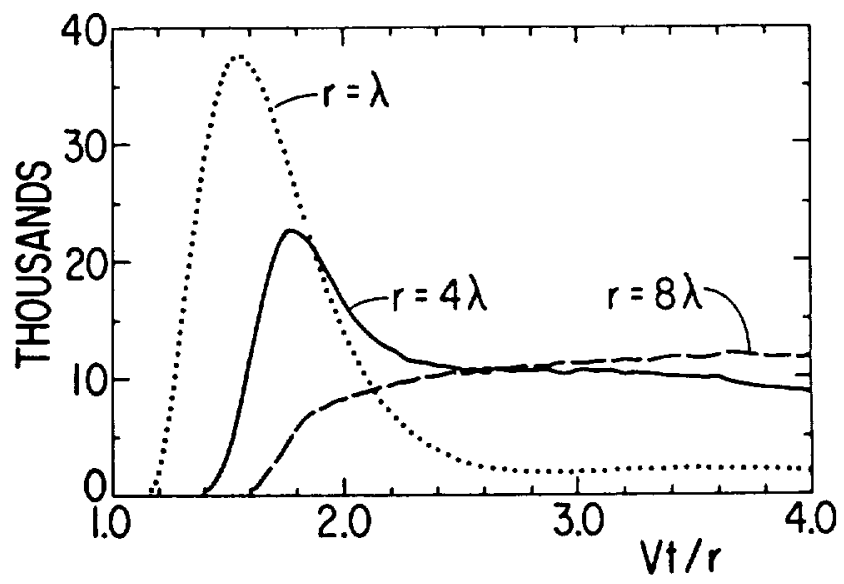

Figure 5. Profiles of intensity vs. time at a fixed distance.

Observations from spacecraft generally take the form of profiles of intensity vs. time at an essentially fixed position in space. In Figure 5, results from MPP simulations, which are presented in this form, show what happens when the coherent pulse sweeps over an observer at a fixed location. The dotted curve, which refers to a mean free path equal to the distance of the observer from the sun, defines a peak followed by a very small wake. As the mean free path becomes smaller, this pulse becomes less prominent relative to the wake ( solid curve), until it is 
submerged in the onset ( dashed curve ). Examples of all three of these behaviors are seen among observed solar particle events, along with events whose extremely slow onset can be understood in terms of standard diffusion theory. Consequently, the MPP results make it clear that all types of prompt solar particle events, diffusive, abrupt onset and coherent, can be understood as morphological stages in a continuous sequence of profiles predicted for increasing mean free paths by the theory of charged particle transport. The same basic physics explains all types.

\section{ANGULAR DISTRIBUTIONS}

The discussion above has focused on isotropic intensities in which particles are counted regardless of their directions. In fact both predicted and observed intensities are often highly anisotropic, and valuable information can be derived from a careful analysis of these anisotorpies. To illustrate this point, Figure 6 presents numbers of particles binned according to $\mu$ and summed over a coherent pulse. Evidently, the intensity in the forward hemisphere $\mu>0$ is overwhelming larger than that in the backward hemisphere. The solid curve which gives the result of a MPP simulation is in very good agreement with the dashed curve, which gives the function $\exp (G)$ which is expected from theory.

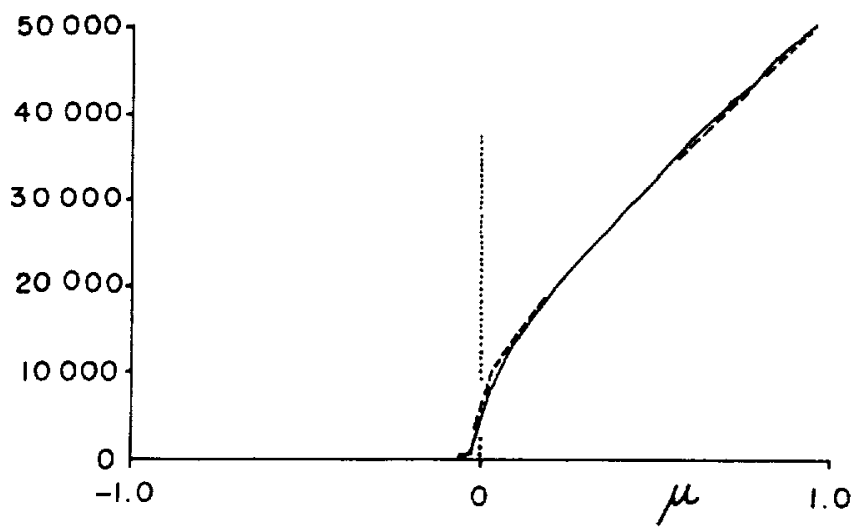

Figure 5. Angular distributions for particles summed over the coherent pulse.

\section{CONCLUSIONS}

Results obtained on the MPP with the aid of Monte Carlo methods are equivalent in every detail to those based upon careful use of more traditional methods, but they are less subject to error and are closer to the physics. These characteristics offer tremendous advantages in the investigation of exotic transport regimes for which no theoretical description is available. In particular, the formulation of problems in which particles gain or lose energy leads to prohibitively large conventional computations, but their Monte Carlo versions are not significantly more complicated than the one described here. We intend to exploit these advantages in the investigation of two such problems: Adiabatic deceleration of cosmic-rays due to expansion of the solar-wind, and the loss of energy by electrons in radio sources due to synchrotron radiation. On a more immediate time scale, the current simulations, which embody an accurate and useful description of solar particle events, will be applied to the interpretation of observations.

\section{ACKNOWLEDGEMENTS}

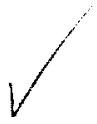

This research was supported by NASA under Grant NGR-21-002-066.

\section{REFERENCES}

1. Lin, R. P., "Emission and Propagation of $\sim 40 \mathrm{keV}$ Solar Flare Electrons," Solar Physics, 15, 453, 1970.

2. Case, K. M., and Zweifel, P. F., "Linear Transport Theory", Addison-Wesley, Reading MA, 1967.

3. Earl, J. A., "Coherent Propagation of Charged Particle Bunches in Random Magnetic Fields", Astrophysical Journal, 188, 379, 1974.

4. Earl, J. A., "Analytical Description of Charged Particle Transport Along Arbitrarary Guiding Field Configurations", Astrophysical Journal, 251, 739, 1981.

5. Earl, J. A., "Simulation of Charged Particle Transport on the Massively Parallel Processsor", First Symposium on th Frontiers of Massively Parallel Scientific Computation, NASA Goddard Space Flight Center, Sept. 24 - 25, 1986

6. Earl, J. A., "Charged Particle Transport Calculations on the Massively Parallel Processor", Conference Papers. 20 th International Cosmic Ray Conference, Moscow, 3, 198,1987

7. Jokipii, J. R., "Cosmic-Ray Propagation I, Charged Particles in a Random Magnetic Field", Astrophysical Journal, 146, 480, 1966.

8. Kota, J., Merenyi, E., Jokipii, J. R., Gombosi, T. I., and Owens, A. J., "A Numerical Study of the Pitch-Angle Scattering of Cosmic-Rays", Astrophysical Journal, 254, 398, 1982 . 